\title{
Perspectivas de integralidade na percepção de profissionais do Núcleo Ampliado de Saúde da Família e Atenção Básica em Belém do Pará, Brasil
}

\author{
Perspectives of integrality in perception of professionals of Expanded Nucleus of Family \\ Health and Basic Care in Belém, Pará, Brazil
}

Perspectivas de integralidad en la percepción de profesionales del Núcleo Ampliado de Salud de la Familia y Atención Básica en Belém do Pará, Brasil

Thaís do Socorro da Luz Silva ${ }^{1 *}$, Marcos Valério Santos da Silva.

\begin{abstract}
RESUMO
Objetivo: Analisar o Núcleo ampliado de Saúde da Família (Nasf-AB) quanto a integralidade, sob a perspectiva de profissionais de duas equipes Nasf-AB no município de Belém, Estado do Pará, Brasil. Métodos: Tratou-se de uma abordagem qualitativa do tipo estudo de caso com observação participante. Foram realizadas entrevistas semiestruturadas com nove profissionais de duas equipes Nasf-AB. A entrevista foi guiada por um roteiro com questões divididas em duas partes, a primeira compreendeu informações sobre caracterização profissional e a segunda teve questões referentes as práticas de integralidade. Posteriormente foi feita a análise de conteúdo desse material, no período de Agosto a Dezembro de 2017. Resultados: Os resultados revelam que cinco dos profissionais possuem pós-graduação, mas apenas um possui especialização na área de Saúde Coletiva, indicando formação teórica específica insuficiente da equipe. Parte dos entrevistados caracterizou a integralidade como assistência geral, visão do indivíduo como um todo e também como integração no processo de trabalho. Conclusão: Os profissionais demonstraram compreender a essência do princípio da integralidade de maneira crítica, enquanto bandeira de luta para modificação das práticas de saúde.
\end{abstract}

Palavras-chave: Integralidade em saúde, Atenção primária à saúde, Estratégia saúde da família.

\begin{abstract}
Objective: Analyze the extended Family Health Center (Nasf-AB) regarding integrality, from the perspective of professionals from two Nasf-AB teams in Belém, Pará, Brazil. Methods: This was a qualitative approach of the case study type with participant observation. Semi-structured interviews were carried out nine professionals from two Nasf-AB teams. The interview was guided by a script with questions divided into two parts, the first comprised information about professional characterization and the second had questions regarding integrality practices. Subsequently, a content analysis of this material was carried out, from August to December 2017. Results: The results reveal that five of the professionals have a postgraduate degree, but only one has a specialization in the area of Health Collective, indicating specific theoretical training specific to team. Part of the interviewees characterized the integrality as general assistance, vision of the individual as a whole and also as integration in the work process. Conclusion: Practitioners demonstrated a critical understanding of the essence of the integrality principle, as a banner of struggle to modify health practices.
\end{abstract}

Key words: Integrality in health, Primary health care, Family health strategy.

\section{RESUMEN}

Objetivo: Analizar el Centro de Salud de la Familia ampliado (Nasf-AB) em relación a la integralidad, desde la perspectiva de los profesionales de dos equipos Nasf-AB en Belém, Estado de Pará, Brasil. Métodos: Este fue um enfoque cualitativo del tipo estudio de caso con observación participante. Se realizaron entrevistas semiestructuradas a nueve profesionales de dos equipos Nasf-AB. La entrevista fue guiada por un guión con

\footnotetext{
${ }^{1}$ Universidade Federal do Pará (UFPA), Belém - PA. *E-mail: ft.thaisluz@yahoo.com.br
} 
preguntas divididas en dos partes, la primera contenía información sobre caracterización profesional y la segunda contenía preguntas sobre prácticas de integralidad. Posteriormente, se realizó un análisis de contenido de este material, em el período de agosto a diciembre de 2017. Resultados: Los resultados revelan que cinco de los profesionales poseen posgrado, pero solo uno posee especialización en el área de Salud Colectiva, lo que indica una formación teórica específica para equipo. Parte de los entrevistados caracterizó la integralidad como asistencia general, visión del individuo como un todo y también como integración en el proceso de trabajo. Conclusión: Los profesionales demostraron uma comprensión critica de la esencia del principio de la integralidad, como bandera de lucha por modificar las prácticas de salud.

Palabras clave: Integralidad en salud, Atención primaria a la salud, Estrategia de salud de la familia.

\section{INTRODUÇÃO}

O Sistema Único de Saúde (SUS) abrange um conjunto organizado e articulado de serviços e ações de saúde do Estado brasileiro. Surgiu como uma proposta inovadora de promover a oferta de serviços públicos a todos os cidadãos. Sendo considerado um sistema complexo por articular e coordenar ações promocionais e de prevenção, com as de cura e reabilitação (VASCONCELOS CM e PASCHE DF, 2006).

O SUS possui diretrizes que são a universalidade do acesso, a integralidade, o cuidado organizado em rede e a equidade da oferta e das oportunidades em saúde (BRASIL, 2010). Nesse contexto surge a concepção de integralidade, essa diretriz garante ao usuário uma atenção que abrange as ações de promoção, prevenção, tratamento e reabilitação, com garantia de acesso a todos os níveis e complexidades do SUS (BRASIL, 2010).

Neste sentido, desde a sua criação na constituição de 1988, o SUS vem mostrando significativos avanços, entre os quais a criação de programas que visam à reorientação do modelo assistencial vigente na época, que possuía como base a atenção curativa com postura medicalizante, verticalizada e individualista, centrada na atuação do médico, onde havia clara dicotomia entre assistência e prevenção (BRASIL, 2010; ROMAGNOLI, 2009; SANTOS MLM, et al, 2016).

Na década de 1990, iniciou a implantação do Programa Saúde da Família (PSF) pelo MS, com o objetivo de reorganizar as ações de atenção primária no país e substituir o modelo tradicional, levando a saúde para mais perto das famílias e pautada na prevenção e promoção de saúde (BRASIL, 2008; BRASIL, 2010; STARFIELD B, 2004). A partir de 1997, passou a ser considerada pelo MS como estratégia de mudança do modelo assistencial, chamado Estratégia Saúde da Família (ESF) (BRASIL, 2010; PAIM JS, 2003), sendo adotado como modelo prioritário de reorganização da atenção primária em saúde no país (BRASIL, 1997).

Dessa forma, o Ministério da Saúde criou em 2008 os Núcleos de Apoio à Saúde da Família (Nasf) visando apoiar a inserção das equipes de Saúde da Família (SF) na rede de serviços, ampliando assim a abrangência, a resolutividade, a territorialização e a regionalização das ações da atenção básica no Brasil, (BRASIL, 2008; BRASIL, 2010; NASCIMENTO CMB, 2014). A partir da PNAB (2017) esses núcleos passam a ser denominados de Núcleo Ampliado de Saúde da Família e Atenção Básica (Nasf-AB) (BRASIL, 2017).

O Nasf-AB é constituído por uma equipe multiprofissional, para atuarem no apoio e em parceria com as equipes de SF. Logo os profissionais do Nasf-AB atuam em suas especialidades para complementar a ação das equipes de SF. Sua função é, conjuntamente com as equipes SF a ele vinculados, organizar seu processo de trabalho com foco nos territórios de sua responsabilidade (BRASIL, 2010; SOUZA FLD, et al., 2013).

O Nasf-AB, portanto, faz parte da Atenção Básica $(A B)$, porém não se institui como um serviço com espaço físico independente. Isto é, os profissionais do núcleo utilizam-se do próprio espaço das unidades básicas de saúde e desenvolvem o seu trabalho no mesmo território adstrito. Atuando de forma integrada à Rede de Atenção à Saúde e seus serviços, a partir de demandas identificadas no trabalho conjunto com as equipes vinculadas (BRASIL, 2014).

O Núcleo ampliado possui como compromisso atuar na promoção de mudanças de atitude e na ação dos profissionais da ESF e entre sua própria equipe Nasf-AB, abrangendo a atuação de ações intersetoriais e 
interdisciplinares, promoção, prevenção e reabilitação da saúde e cura, assim como humanização de serviços, educação permanente dos profissionais e da população, promoção da integralidade, participação social, educação popular e a organização territorial dos serviços de saúde (BRASIL, 2010).

A integralidade pode ser conhecida como a principal diretriz a ser praticada pelo Nasf-AB e a sua busca nos serviços de saúde deve ser, portanto, um processo em construção, sendo a equipe de SF um fértil campo ao fomento da atenção integral (BRASIL, 2010). Este processo se efetiva na medida em que são consideradas, no processo de cuidado, as ações de promoção da saúde, acompanhamento e monitoramento dos indivíduos em seus diversos aspectos (PAGANI R e NASCIMENTO DDG, 2016).

$O$ objetivo da pesquisa foi analisar o Nasf-AB no contexto da integralidade, sob a perspectiva de profissionais de duas equipes Nasf-AB no município de Belém Estado do Pará.

\section{MÉTÓDOS}

O projeto de pesquisa foi realizado respeitando as Normas de Pesquisa Envolvendo Seres Humanos Resolução 510/2016 do Conselho Nacional de Saúde, após aceite do orientador. A coleta foi realizada após autorização da instituição envolvida na pesquisa e após a aprovação do Comitê de ética em pesquisa do Hospital Universitário João de Barros Barreto (HUJJB) por meio do protocolo 2.070.728 e CAAE 65993617.7.0000.0017.

O presente estudo trata-se de uma abordagem qualitativa, do tipo estudo de caso, com observação participante, ou seja, o pesquisador se inseriu no interior do grupo observado interagindo com os sujeitos em duas equipes da ESF e NASF do município de Belém Estado do Pará.

Foram incluídos na pesquisa os profissionais do Nasf-AB I e Nasf-AB II colaborativos e que aceitaram participar voluntariamente da pesquisa. Adotou-se como critério de exclusão, participantes que se encontravam de férias, licença ou ausentes após três tentativas de entrevistas. Foram entrevistados nove participantes, sendo três profissionais da equipe Nasf-AB I e seis profissionais da equipe Nasf-AB II.

A entrevista foi semiestruturada e guiada por um roteiro com questões divididas em duas partes, a primeira compreendeu informações sobre caracterização em relação à idade, sexo, ano de conclusão de graduação, titulação, tempo de experiência profissional para os profissionais e a segunda teve questões referentes as práticas de integralidade.

As entrevistas foram realizadas individualmente com os profissionais, previamente agendada de acordo com dia, horário e local com sala exclusiva disponibilizada na USF e UMS. Cada entrevista teve duração média de 20 minutos. O conteúdo das entrevistas foi gravado em áudio com consentimento dos participantes e transcrito na íntegra para evitar possíveis danos ao mesmo e posteriormente foram analisados.

O acompanhamento foi realizado no período de Agosto a Dezembro de 2017, sendo os três primeiros meses a coleta foi realizada na unidade de Estratégia Saúde da Família I (ESF I) e os dois últimos meses na unidade Estratégia Saúde da Família II (ESF II). Para a análise desse material, inicialmente realizou-se a leitura completa e exaustiva do mesmo. As falas presentes nas respostas do questionário foram identificadas por E1, E2 e assim sucessivamente para resguardar a identidade dos sujeitos da pesquisa, e em seguida formamos as categorias emergentes das falas dos participantes.

\section{RESULTADOS E DISCUSSÃO}

Constatou-se que as duas equipes Nasf-AB possuíam no total 11 (onze) profissionais, representantes de distintas profissões (terapeuta ocupacional, psicólogo, assistente social, fisioterapeuta, educador físico, nutricionista e fonoaudiólogo). Sendo que destes nove participaram da pesquisa, pois dois profissionais da equipe Nasf-AB I estavam ausentes no serviço no período da coleta de dados, uma encontrava-se de licença maternidade e o outro de férias. Outras características podem ser observadas na tabela Tabela 1. 
Tabela 1 - Caracterização dos profissionais das equipes Nasf-AB I e Nasf-AB II do município de Belém do Pará, 2017.

\begin{tabular}{ccccc}
\hline Sexo & Idade & Graduação & $\begin{array}{c}\text { Tempo de conclusão da } \\
\text { graduação (Anos) }\end{array}$ & $\begin{array}{c}\text { Tempo de experiência } \\
\text { profissional (Anos) }\end{array}$ \\
\hline F & 43 & Psicologia & 20 & 3 \\
F & 39 & Serviço Social & 11 & 3 \\
F & 28 & Fonoaudiologia & 5 & 3 \\
F & 28 & Fisioterapia & 7 & 3 \\
F & 34 & Nutrição & 3 & 1 \\
M & 56 & Educação Física & 13 & 2 \\
F & 35 & Terapia Ocupacional & 13 & 3 \\
M & 30 & Terapia Ocupacional & 5 & 2 \\
F & 37 & Fonoaudiologia & 6 & 3 \\
\hline
\end{tabular}

Fonte: Silva TSL e Silva MVS, 2021.

A maioria dos profissionais é do sexo feminino, sendo em número de sete (77,78\%); a idade mínima apresentada foi vinte e oito anos e a máxima cinquenta e seis anos, com média de idade igual a 36,67 anos. O tempo de conclusão da graduação variou de três a vinte anos, com período de experiência profissional na equipe Nasf de um a três anos na equipe (Tabela 1).

No que concerne às categorias profissionais as duas equipes Nasf apresentam: dois terapeutas ocupacionais, dois psicólogos, um educador físico, um fisioterapeuta, um fonoaudiólogo, um nutricionista e um assistente social.

Quanto à formação profissional, apenas um entrevistado possui especialização na área de saúde coletiva, sendo que quatro possuem especialização em outras áreas e quatro não possuem especialização (Tabela 2).

Tabela 2 - Características dos entrevistados a nível de titulação, Belém do Pará 2017.

\begin{tabular}{cccc}
\hline Entrevistado & Graduação & $\begin{array}{c}\text { Especialização } \\
\text { Saúde coletiva }\end{array}$ & $\begin{array}{c}\text { Especialização outras } \\
\text { áreas }\end{array}$ \\
\hline E1 & Nutrição & - & $\begin{array}{c}\text { Nutrição clínica } \\
\text { Desenvolvimento infantil }\end{array}$ \\
E3 & Terapia Ocupacional & - & - \\
E4 & Fonoaudiologia & - & Psicologia Clínica \\
E5 & Psicologia & - & - \\
E6 & Serviço Social & - & - \\
E7 & Terapia Ocupacional & Saúde Pública & Audiologia \\
E8 & Fonoaudiologia & - & - \\
E9 & Educação Física & - & - \\
\hline
\end{tabular}

Fonte: Silva TSL e Silva MVS, 2021.

O baixo índice de profissionais com especialização na área de APS e áreas correlatas foi encontrado em outras pesquisas científicas (CASTRO RCL, et al., 2012; SILVA AS, et al., 2014). De acordo Silva SA et al. (2014), isso pode estar relacionado com a rotatividade de profissionais. Esta alta rotatividade está associada a vínculos precários de trabalho, à ausência de capacitação adequada, ao tempo de locomoção e à falta de materiais para a realização do trabalho.

Em contrapartida na pesquisa de Reis ML, et al. (2016), que buscou compreender os significados atribuídos da equipe multiprofissional ao trabalho desenvolvido em uma unidade do Nasf-AB, observou-se 
que entre os 14 profissionais, 50\% dos quais possuíam especialização em Saúde da Família, apenas dois participantes não apresentavam pós-graduação. Os autores concluíram que a qualificação profissional trouxe um diferencial significativo à assistência. Outras pesquisas encontradas na literatura também mostraram que os profissionais mais qualificados avaliam melhor a APS (CASTRO RCL, et al., 2012; CHOMATAS E, et al., 2013).

Nesse sentido é importante conhecer o perfil dos profissionais que atuam no Nasf-AB, para ser possível compreender o processo de formação profissional e se estes estão capacitados a atuar na atenção primária. Diante disso, proporcionar ferramentas que incentivem a qualificação e, consequentemente, a melhoria na construção das práticas assistenciais.

A segunda parte da pesquisa tratou-se de interrogar os informantes sobre o conhecimento acerca da integralidade na atenção à saúde. Percebeu-se uma variedade de sentidos, parte dos entrevistados a caracterizaram como assistência geral, visão do indivíduo como um todo e também como integração no processo de trabalho.

"Olha, desde a lei do SUS, a Lei 8080 - ela diz que a integralidade é universalidade da Saúde. Então, a pessoa tem que ter a assistência geral, social, médica, psicológica e de lazer. Isso tudo na lei está. O NASF ele é parecido com isso porque ele pega assim as profissões. Então ele chega perto dessa integralidade" (E4).

A integralidade foi considerada como assistência geral para o entrevistado E4, pois em sua percepção a integralidade está associada à visão integral do ser humano. Para esse profissional o termo envolve o atendimento de forma universal, levando em consideração a integridade física, social e mental. Apesar de usar o termo "universalidade" no contexto da integralidade essa fala se aproxima com dois sentidos atribuídos a este princípio, a abordagem integral do indivíduo e a abordagem multiprofissional.

A integralidade não visa reduzir o usuário ao sistema biológico ou a queixa que provoca a dor. Apresenta um sentido que se relaciona com uma atitude dos médicos que seria desejável, caracterizada como um traço de boa medicina, ou seja, o uso prudente dos conhecimentos sobre a doença, mas guiado por uma visão abrangente das necessidades dos sujeitos tratados (MATTOS RA, 2009).

Segundo Mattos RA (2009) uma atitude que pode ilustrar um dos sentidos de integralidade ligados aos ideais da medicina integral é o médico que, no encontro com o paciente, busca reconhecer fatores de riscos de outras doenças que não são relacionadas no sofrimento real daquele paciente, e/ou investiga a presença de doenças que ainda não se expressaram em dor.

Corroborando com esse pensamento Viegas SMF e Penna CMM (2015), caracterizam essa dimensão da integralidade na expressão "olhar a pessoa", concretizando a abordagem do usuário como sujeito integrado à família, ao domicílio e à comunidade, ou seja, em sua vivência no mundo.

O Nasf-AB possui como fundamento ideológico a integralidade do cuidado aos usuários, como uma visão ampliada da clínica, ou seja, a saúde depende de fatores além dos biológicos. Assim, a atuação das equipes profissionais deve levar em conta essa pluralidade de fatores para intervir (QUEVEDO MP e OLIVEIRA FR, 2016).

O cuidado em saúde deve contrapor-se a abordagem fragmentada e reducionista dos indivíduos, para isso os profissionais de saúde têm a necessidade de apreender este usuário em sua dimensão biopsicossocial (QUEVEDO MP e OLIVEIRA FR, 2016).

Deste modo, o depoimento do entrevistado E4 demonstra concordância com esses autores ao mencionar que a pessoa necessita de assistência geral, que abrange as áreas: social, médica, psicológica e de lazer, isto é, o indivíduo deve ser visualizado como um todo e que várias condições sejam elas de moradia, desemprego, violência, entre outras podem afetar a qualidade de vida familiar e comunitária.

Outro sentido da integralidade está voltado ao modo de organizar as práticas de saúde e a articulação entre a rede de assistência para garantir acesso e resolutividade às questões de saúde. Na fala abaixo visualiza-se esses dois sentidos abordados. 
"A integralidade eu vejo em dois aspectos. A integralidade, por exemplo, assistencial acontece no processo de trabalho entre o Nasf e entre as estratégias, os outros serviços de atenção primária. Essa primeira integralidade assistencial está de acordo com o conceito de saúde e serve para todos os profissionais da saúde. [...] E tem a integralidade assistencial que vejo assim, você poder ter o ponto de coordenação que a ESF e ter informações em todos os pontos que o paciente for encaminhado" (E6).

"Eu acho assim que a integralidade [...] é você conseguir integrar todos os sistemas de saúde estratégia, unidade básica, Nasf para tentar fazer com que esse indivíduo não tenha doença. Nem sempre a questão da prevenção e orientação tá” (E3).

O processo de trabalho entre a equipe Nasf e os outros serviços de atenção primária foi um ponto destacado pelos entrevistados E6 e E3. O primeiro relato caracterizou esse termo também na dimensão da integração e a organização dos serviços de saúde.

Esses dados encontram ressonância na pesquisa de Miranda GBN (2016) realizada também com profissionais das equipes ESF e Nasf-AB na capital paraense, evidenciando relação deste termo à visão integral do ser humano e também na perspectiva da articulação dos serviços.

Para Quevedo MP e Oliveira FR (2016) o modo como as práticas de saúde são articuladas no cotidiano de trabalho impacta diretamente a integralidade da atenção ofertada aos usuários. Assim sendo, o Nasf-AB deve trabalhar no sentido de discutir junto aos serviços a maneira como os mesmos devem ser organizados, para poderem apreender de forma ampliada as necessidades da população a ser cuidada, sejam essas necessidades explícitas ou não.

Mattos RA (2009) aponta o termo relacionado à integralidade como modo de organizar as práticas, onde os serviços devem ser organizados para realizar uma compreensão ampliada das necessidades da população que atendem. Ou seja, que os serviços não estejam organizados exclusivamente para responder às doenças de uma população, embora devam responder a tais doenças.

Segundo Quevedo MP e Oliveira FR (2016) o Nasf-AB deve organizar o processo de trabalho para garantir o acesso da população a cuidados de saúde de acordo com a rede de serviços disponíveis no território, considerando ações de caráter assistencial e curativo, bem como atividades de promoção e prevenção, de modo individual e coletivo, por meio de ações locais, intersetoriais e clínicas.

No depoimento a seguir são apresentadas algumas dificuldades à integralidade nos serviços tais como, a comunicação inadequada entre os níveis de assistência, ausência de contrarreferências e de seguimento. Além disso, notou-se um sentimento de desânimo do participante, ao mencionar que não possui informações dos usuários quando são encaminhados para outros serviços.

"Eu entendo que cada profissional que está inserido no contexto da atenção básica, eles deveriam se unir, se integrar mais para que o serviço possa vim realmente a ter um êxito. Porque às vezes a gente faz o encaminhamento, por exemplo, lá para a casa mental, o paciente precisa desse atendimento e foi para lá, a gente não tem esse retorno do paciente. A gente não sabe se esse paciente foi atendido, o que aconteceu com ele, se está sendo atendido, acompanhado. A gente não tem, até porque o paciente não volta mais para a gente. Então eu acho que a gente devia se integrar mais dentro das redes de atendimento para o atendimento realmente funcionar" (E5).

Resultados semelhantes foram encontrados na pesquisa de Macedo LM e Martin STF (2014), onde foi identificado que as dificuldades de integração das ações da Unidade Básica com os demais níveis de cuidado foram destacadas com conotação afetiva negativa, indicando indignação, descrédito, desânimo e impotência frente às limitações na resolubilidade das demandas.

Os dados corroboram também com a pesquisa de Oliveira IF et al. (2017), onde os principais desafios assinalados pelos entrevistados para as articulações com a rede foram as dificuldades de comunicação entre 
as instituições; a falta de comprometimento ou disponibilidade para parcerias; e a descontinuidade dos atendimentos encaminhados pela equipe Nasf-AB para a rede, não havendo responsabilidade compartilhada pelo cuidado ao usuário.

Para esse mesmo autor é necessário que os profissionais e equipes desenvolvam um trabalho articulado com redes e instituições que estão fora do seu próprio setor, a fim de superar a fragmentação dos conhecimentos e das estruturas sociais, produzindo efeitos mais significativos na saúde da população e reestruturando a lógica de atendimento (OLIVEIRA IF et al., 2017).

A integralidade também é considerada como uma prática que visa atender a população do território adscrito pela estratégia "da melhor forma, mas nas suas especificidades". Ou seja, neste discurso há o reconhecimento de que as pessoas possuem suas peculiaridades.

"A integralidade, eu entendo que é a gente poder atender a todos ali do nosso território, que a estratégia tá, da melhor forma, mas nas suas especificidades. Eu acho que foi, por isso que houve a mudança que justamente para gente atender cada especificidade, cada grupo, porque apesar de ser a mesma população, cada um tem necessidade diferente, cada grupo tem necessidades diferentes. Então, eu entendo que é isso. É atender a todos, mas nas suas especificidades" (E2).

Conforme Viegas SMF (2010) apesar de ser difícil a prática da integralidade com o tempo pode-se identificar a quem dar mais atenção ou as demandas específicas. Neste sentido, essa identificação deveria implicar nas respostas as desigualdades, a equidade nas ações.

Portanto a equidade postula a diferença. Todo cidadão é igual perante o SUS e será atendido segundo suas necessidades que são singulares e específicas a cada um. Em cada população, existem grupos que vivem de forma diferente, decorrentes da diversidade do modo de viver, de adoecer e de ter oportunidade de satisfazer suas necessidades de vida. Reconhece desse modo o imperativo de tratar desigualmente os desiguais, com implicações importantes para a questão da demanda (SPINK MJP, 2007).

\section{CONCLUSÃO}

Buscou-se compreender a construção das práticas de integralidade em saúde no contexto do Nasf-AB, com esse intuito procurou-se conhecer o que a equipe entendia por integralidade em saúde e por meio disso observar como eles aplicam esse conceito em seu cotidiano nas unidades. De modo geral foi observado que a essência do princípio da integralidade foi compreendida de maneira crítica pelos participantes do estudo, enquanto bandeira de luta para modificação das práticas de saúde e consolidação do modelo assistencial.

\section{REFERÊNCIAS}

1. BRASIL. Ministério da Saúde. Secretaria de Atenção à Saúde. Portaria № 2.436, de 21 de Setembro de 2017. Estabelece a revisão de diretrizes para organização da Atenção Básica. Política Nacional de Atenção Básica / Ministério da Saúde. Secretaria de Atenção à Saúde. Departamento de Atenção Básica. Brasília: Ministério da Saúde, 2017; 38p.

2. BRASIL. Ministério da Saúde. Secretaria de Atenção à Saúde. Departamento de Atenção Básica. Núcleo ampliado à Saúde da Família. Brasília: Ministério da Saúde, 2014; 39: 116.

3. BRASIL. Ministério da Saúde. Secretaria de Atenção à Saúde. Departamento de Atenção Básica. Diretrizes do Nasf: Núcleo de Apoio à Saúde da Família. Brasília: Ministério da Saúde, 2010.

4. BRASIL. Ministério da Saúde. Portaria $n^{\circ} .154$, de 24 de Janeiro de 2008. Cria os Núcleos de Apoio à Saúde da Família NASF. Diário Oficial da República Federativa do Brasil, 2008.

5. BRASIL. Ministério da Saúde. Secretaria de Assistência à Saúde. Coordenação de Saúde da Comunidade. Saúde da Família: uma estratégia para a reorientação do modelo assistencial. Brasília: Ministério da Saúde. 1997.

6. CASTRO RCL, et al. Avaliação da qualidade da atenção primária pelos profissionais de saúde: comparação entre diferentes tipos de serviços. Cadernos de Saúde Pública. 28(9):1772- 84, 2012.

7. CHOMATAS E, et al. Avaliação da presença e extensão dos atributos da atenção primária em Curitiba. Revista Brasileira Med Fam Comunidade. 8(29):294-303. 2013.

8. MACEDO LM, MARTIN STF. Interdependência entre os níveis de atenção do Sistema Único de Saúde (SUS): significado de integralidade apresentado por trabalhadores da atenção primária. Interface (Botucatu). 18(51):647-659. 2014. 
9. MATTOS RA. Os sentidos da integralidade: algumas reflexões acerca de valores que merecem ser definidos. IN: PINHEIRO R, MATTOS RA. Os sentidos da integralidade na atenção e no cuidado à saúde. 8ª ed. Rio de Janeiro: UERJ, IMS: ABRASCO, 2009; p. 43-68.

10. MIRANDA GBN. Integralidade no trabalho em saúde: dificuldades, possibilidades e perspectivas gerais da abordagem multiprofissional na visão dos trabalhadores de uma equipe da Atenção Primária de Belém-Pará. Dissertação (Mestrado em Saúde, Ambiente e Sociedade) - Programa de Pós-graduação em Saúde, Ambiente e Sociedade na Amazônia da Universidade Federal do Pará, Belém - Pará, 2016; 231p

11. NASCIMENTO CMB. A organização e desenvolvimento da atenção à saúde pelo núcleo de apoio à saúde da família. 182p. Tese (Doutorado em Saúde Pública) - Centro de Pesquisas Aggeu Magalhães. Fundação Oswaldo Cruz. Recife, 2014.

12. OLIVEIRA IF, et al. A atuação do psicólogo nos Nasf: desafios e perspectivas na atenção básica. Temas em Psicologia, 2107; 25(1).

13. PAGANI R, NASCIMENTO DDG. Equipe do Núcleo de apoio à saúde da família: criar e recriar as possibilidades do seu fazer no cotidiano da saúde. In: SANTOS MLM, et al. O Nasf em cena: tecnologias e ferramentas de trabalho no cotidiano das equipes. Edição temática Nasf. Porto Alegre: Rede Unida, 2016; p. 53-83.

14. PAIM JS. Modelos de Atenção e Vigilância da Saúde. In: ROUQUARYOL MZ, ALMEIDA FILHO N. Epidemiologia e Saúde. Rio de janeiro: Medsi, 2003; p. 567-586.

15. QUEVEDO MP, OLIVEIRA FR. O cuidado como prática reflexiva. In: SANTOS MLM, et al. O Nasf em cena: tecnologias e ferramentas de trabalho no cotidiano das equipes. Edição temática Nasf. Porto Alegre: Rede Unida, 2016; p.15-49.

16. REIS ML, et al. Avaliação do trabalho multiprofissional do núcleo de apoio à saúde da família (NASF). Revista Texto Contexto Enfermagem, 2016; 25(1).

17. ROMAGNOLI RC. Breve estudo institucionalista acerca do Programa de Saúde da Família. Saúde Soc. [online], 2009; 18(3): 525-536.

18. SANTOS MLM, et al. O Nasf-AB em cena: tecnologias e ferramentas de trabalho no cotidiano das equipes. Edição temática Nasf-AB. Porto Alegre: Rede Unida, 2016; p.178.

19. SILVA SA. Avaliação da atenção primaria à saúde: visão dos profissionais de saúde. Revista Esc Enfermagem USP, 2014; 48(esp): 126-132.

20. SOUZA FLD. Implantação do Núcleo de Apoio à Saúde da Família: percepção do usuário. Revista Saúde em Debate, 2013; 37(97): 233-240.

21. SPINK MJP. Sobre a possibilidade de conciliação do ideal da integralidade nos cuidados à saúde e a cacofonia da demanda. Saúde e Sociedade, 2007; 16(1): 18-27.

22. STARFIELD B. Atenção primária: equilíbrio entre necessidades de saúde, serviços e tecnologia. Brasília: UNESCO, Ministério da Saúde, 2020; p. 726.

23. VASCONCELOS CM, PASCHE DF. O Sistema Único de Saúde. In: CAMPOS GWS, et al. Tratado de Saúde Coletiva. São Paulo/ Rio de Janeiro: Ed. Hucited/ Ed. Fiocruz, 2006.

24. VIEGAS SMF, PENNA CMM. As dimensões da integralidade no cuidado em saúde no cotidiano da estratégia saúde da família no vale do Jequitinhonha, MG, Brasil. Revista Interface Comunicação Saúde Educação, 2015; 19(55): 10891100.

25. VIEGAS SMF. A integralidade no cotidiano da estratégia saúde da família em municípios do vale do Jequitinhonha. Tese (Doutorado em enfermagem). Escola de Enfermagem. Universidade Federal de Minhas Gerais, Belo Horizonte, 2010. 